\title{
Coastline Detection from SAR Images by Level Set Model
}

\author{
Maria Mercede Cerimele ${ }^{1}$, Luigi Cinque ${ }^{2}$, Rossella Cossu ${ }^{1}$, \\ and Roberta Galiffa ${ }^{1}$ \\ 1 Istituto per le Applicazioni del Calcolo "M. Picone" CNR, Roma \\ 2 Universitá degli Studi "Sapienza", Roma
}

\begin{abstract}
In this paper we present an innovative and automatic procedure which is used to extract the coastline from SAR (Synthetic Aperture Radar) images by the level set model. This model consists in a PDE (Partial Differential Equation) equation governing the evolution of a curve corresponding to the zero level of a $3 \mathrm{D}$ function, called level set function, until the curve reaches the edge of the region to be segmented. A coastline is the boundary between land and sea masses. Detecting the coastline is of fundamental importance when monitoring various natural phenomena such as tides, coastal erosion and the dynamics of glaciers. In this case SAR images show problems which arise from the presence of the speckle noise and of the strong signal deriving from the rough or slight sea. In fact in the case of heavy sea the signal determines an intensity similar to the one of land, making it difficult to distinguish the coastline.
\end{abstract}

\section{Introduction}

In this paper we present a level set method applied to the segmentation of SAR (Synthetic Aperture Radar) images with the aim of extracting two regions (land and sea) from them. This method, proposed by Osher and Sethian [1, [2, 3, consists in the identification of an area of interest as the zero level set of an implicit function that evolves according to the PDE (partial differential equation) model with an appropriate speed function. This approach has many advantages, for example the contours represented by the level set function can split and merge naturally during the evolution and this allows the topological changes to be controlled.

The interpretation of SAR images is an essential component of not invasive monitoring in many fields such as urban planning, geology, for instance the erosion of coast [4. In particular detecting the coastline from SAR images is a difficult problem because it is associated with the nature of the signal coming from water and land regions. In fact, the signal return coming from the sea can be frequently indistinct from one coming from the land. Nevertheless, the 
presence of speckle, modeled as a strong multiplicative noise, makes the coastline detection a very complicated issue.

The segmentation methods based on edge detection filters developed for SAR images [5], often show edges which may not form a set of closed curves surrounding connected regions. The traditional segmentation techniques of histogram thresholding and region-based need a preprocessing based on speckle reduction. Moreover, the region-growing techniques have the limit of depending on the selection of the starting points.

In recent years, active contours methods, based on the evolution of curves and on a level set approach have been an important tool for solving image segmentation problems. The active contours have been classified as parametric, also called snakes, or geometric depending on their representation and implementation. In particular, parametric active contours are explicitly represented as curves parameterized in a Lagrangian reference, while the geometric active contours are implicitly represented as two-dimensional functions, called level sets that evolve in an Eulerian reference. For SAR images, the parametric active contours were developed in [6], 7], 8].

In general, in order to extract the contour of a region, the snake-model type uses an algorithm that iteratively deforms an initial curve until it reaches the edge of the region to be segmented. However, this model presents several limitations. In fact, since the curve is represented in parametric form, it is discretized by a set of points, so that during the time evolution topological changes are difficult to compute. Moreover, the errors in the representation may be amplified during the numerical computation. Thus, these problems may affect the results of the segmentation process. Compared to the techniques of parametric active contours, the geometric active contours implemented by level set have the significant advantage of allowing natural and numerically stable topological changes.

In this paper we use the evolution of curves through level set to segment SAR images into two distinct classes, land and sea.

In particular a first approach developed is based on the assumption that each region to segment through level set is modeled by a Gamma distribution. In this case an expression of propagation speed of the front is obtained by computing intensity averages of the regions; the method does not need to reduce speckle noise 9].

A second approach takes into account the problem of filtering speckle noise in the image, which was faced with the application of the SRAD (Speckle Reducing Anisotropic Diffusion) technique to SAR image [10] [11.

Finally we propose an integrated procedure obtained by combining the two previous procedures, taking into account the advantages of the two approaches. The paper is organized as follows. In Section 2 level set method is described. In Section 3 speeds computation related to level set method and noise reducing are presented. Section 4 contains the description of proposed integrated procedure. A conclusion is drawn in Section 5 . 


\section{Mathematical Approach}

Let $\mathbf{I}: \Omega \rightarrow \Re^{n}$ be the intensity image function where $\Omega \subset \Re^{2}$.

The goal of image segmentation is to partition $\Omega$ in order to extract disjoint regions from image $\mathbf{I}$ such that they cover $\Omega$.

The boundaries of these regions may be considered as curves belonging to a family in which time evolution is described by a level set equation. The main advantages of using the level set is that complex shaped regions can be detected and handled implicitly.

In order to obtain the governing equation of a front evolution, we consider a family of parameterized closed contours $\gamma(x(t), y(t), t):[0, \infty) \rightarrow \Re^{2}$, generated by evolving an initial contour $\gamma_{0}(x(0), y(0), 0)$.

We underline that in the curves evolution theory the geometric shape of the contour is determined by the normal component of the velocity. Supposing that $\gamma(x(t), y(t), t)$ is a moving front in the image, if we embed this moving front as the zero level of a smooth continuous scalar 3D function $\phi(x(t), y(t), t)$, known as the level set function, the implicit contour at any time $t$ is given by $\gamma(x(t), y(t), t) \equiv$ $\{(x(t), y(t)) / \phi(x(t), y(t), t)=0\}$.

By differentiating respect to $t$ the expression $\phi(x(t), y(t), t)=0$ the equation for the evolution of the level set function may be derived

$$
\frac{\partial \phi(x(t), y(t), t)}{\partial t}+\frac{d x(t)}{d t} \frac{\partial \phi(x(t), y(t), t)}{\partial x}+\frac{d y(t)}{d t} \frac{\partial \phi(x(t), y(t), t)}{\partial y}=0
$$

We require that the level set function has to satisfy the condition $|\nabla \phi(x(t), y(t), t)| \neq 0$ for all $(x(t), y(t)) \in \gamma(x(t), y(t), t)$; this is possible because $\gamma(x(t), y(t), t)$ is a regular curve. Let $\boldsymbol{n} \equiv\left(n_{1}, n_{2}\right)$ be the normal vector to the curve $\gamma(x(t), y(t), t)$ defined as

$$
n_{1}=\frac{\frac{\partial \phi(x(t), y(t), t)}{\partial x}}{|\nabla \phi(x(t), y(t), t)|} \quad n_{2}=\frac{\frac{\partial \phi(x(t), y(t), t)}{\partial y}}{|\nabla \phi(x(t), y(t), t)|}
$$

that is

$$
\frac{\partial \phi(x(t), y(t), t)}{\partial x}=|\nabla \phi(x(t), y(t), t)| n_{1} \quad \frac{\partial \phi(x(t), y(t), t)}{\partial y}=|\nabla \phi(x(t), y(t), t)| n_{2}
$$

substituting in (1)we obtain

$$
\frac{\partial \phi(x(t), y(t), t)}{\partial t}+\left(n_{1} \frac{d x(t)}{d t}+n_{2} \frac{d y(t)}{d t}\right)|\nabla \phi(x(t), y(t), t)|=0
$$

where $\left(n_{1} \frac{d x(t)}{d t}+n_{2} \frac{d y(t)}{d t}\right)$ describes the curve evolution in the normal direction, so that we can write $\left(n_{1} \frac{d x(t)}{d t}+n_{2} \frac{d y(t)}{d t}\right)=\frac{d \boldsymbol{\gamma}(x(t), y(t), t)}{d t}$ the (2) becomes

$$
\frac{\partial \phi(x(t), y(t), t)}{\partial t}+\frac{d \gamma(x(t), y(t), t)}{d t}|\nabla \phi(x(t), y(t), t)|=0
$$


or also

$$
\frac{d \gamma(x(t), y(t), t)}{d t}=-\frac{\partial \phi(x(t), y(t), t) / \partial t}{|\nabla \phi(x(t), y(t), t)|}
$$

In the following another important intrinsic geometric property will be used, that is the curvature of each level set given by

$$
k=-\nabla \cdot\left(\frac{\nabla \phi(x(t), y(t), t)}{|\phi(x(t), y(t), t)|}\right)
$$

and moreover we assume

$$
\frac{d \gamma}{d t}=v
$$

\section{Speed Computation}

As mentioned above, the level set method starts from the definition of an initial curve in the domain of the image. In our case, the initial curve on the SAR images is placed so as to surround the object of interest (land). The evolution of the initial curve is determined by a speed function, which is a fundamental choice to achieve a good segmentation.

In this paper two different speed functions are introduced and compared.

The first uses a speed function based on modeling the intensity of image by a Gamma distribution. The second uses a speed function based on the computation of image gradient. Finally we propose an integrated procedure obtained by combining the two previous functions.

The SAR PRI (Precision Resolution Image) image here analyzed has been acquired during ERS2 mission. In Table 1 some annotations and calibration data are listed.

\subsection{Average-Based Speed}

The goal of the segmentation process in this work is to extract two types of regions representing land and sea $\left.R_{i} \quad i \in\{1,2\}\right)$.

Let be $I(\mathbf{x})$ the SAR image intensity which we model by a Gamma distribution.

After some probabilistic considerations and algebraic manipulations we obtain

$$
v=\frac{d \boldsymbol{\gamma}}{d t}=-\left(\log \mu_{R_{1}}+\frac{I(\mathbf{x})}{\mu_{R_{1}}}-\log \mu_{R_{2}}-\frac{I(\mathbf{x})}{\mu_{R_{2}}}+\lambda k\right)
$$

where a $\lambda k$ is a regularization term, with $\lambda$ a positive real constant and $k$ the mean curvature function defined in Section $2, \mu_{R_{i}}$ is the mean intensity given

$$
\mu_{R_{i}}=\frac{\int_{R_{i}} I(\mathbf{x}) d \mathbf{x}}{a_{R_{i}}}
$$

and where the area $a_{R_{i}}$ is given

$$
a_{R_{i}}=\int_{R_{i}} d \mathbf{x}
$$


Table 1. LOCATION: Sea between the Corsica and Tuscany coasts

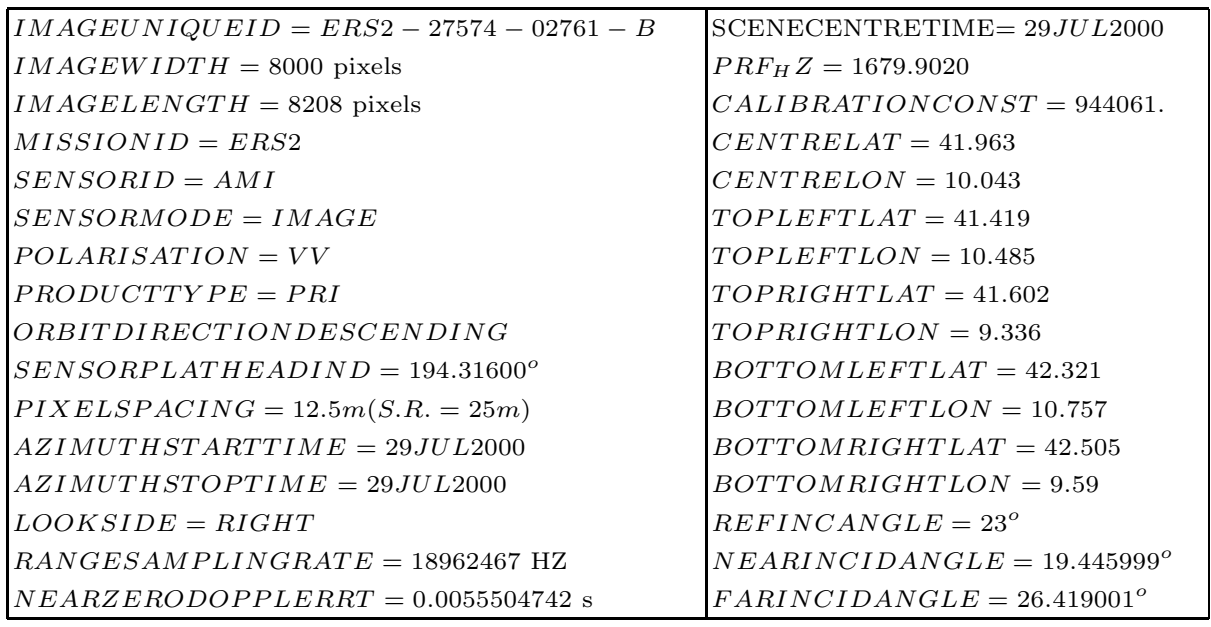

From (3) we obtain the following level set equation

$$
\frac{\partial \phi(x(t), y(t), t)}{\partial t}+v|\nabla \phi(x(t), y(t), t)|=0
$$

We have examined the behavior of the algorithm by using the averaged-based speed. In Figure 1 and Figure 2 the Tests A and B related to two different coastlines are shown: (a) and (b) identify a portion of the initial curves selected (initial data for the algorithm) and (c) and (d) show the respective results obtained when the convergence for the numerical approximation is satisfied (final contours).

From these tests it is evident that the results obtained depend on the initial position of the curve and in particular it has been obtained a better result as the initial curve is located near the coast.

\subsection{Gradient-Based Speed}

In this subsection the image gradient is used to identify the edges or contours. Indeed, if in a zone the value of the gradient is high then the related pixels correspond to an edge. The gradient-based speed function, in this case, is

$$
v=-\frac{1}{1+|\nabla I|^{2}}-\lambda k
$$

where $k$ is the curvature and $\lambda \in(0,1)$ is a constant. Substituting this expression in (3) we obtain the corresponding level set equation. It is well known that in images corrupted by strong noise, the computation of gradient could detect false edges. 


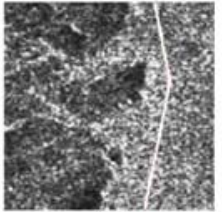

(a)

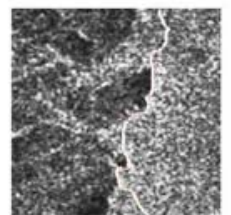

(c)

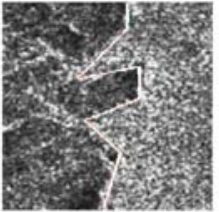

(b)

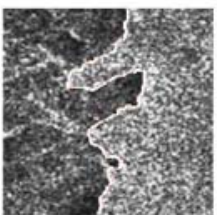

(d)

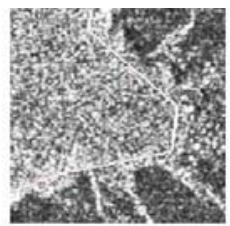

(a)

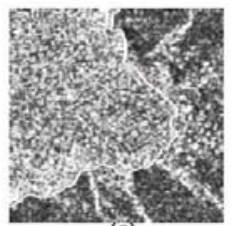

(c)

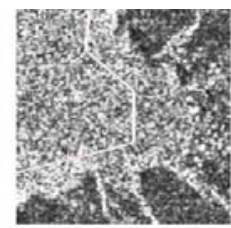

(b)

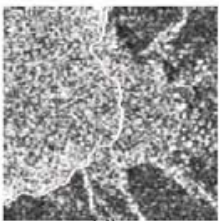

(d)

Fig. 1.

Fig. 2.

Test A: results by average-based speed Test B: results by average-based speed

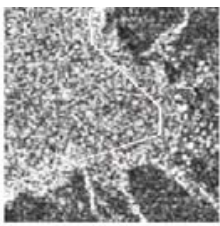

(a)

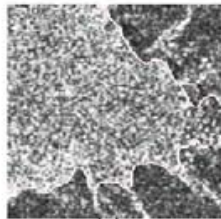

(c)

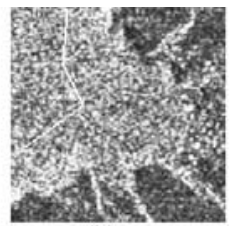

(b)

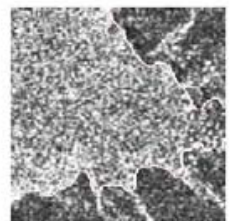

(d)

Fig. 3.

Fig. 4.

Test A: results by gradient based speed Test B: results by gradient based speed

So that, because the SAR images are affected by speckle noise, they are preprocessed by means of the SRAD algorithm (Speckle Reducing Anisotropic Diffusion) which is an extension of Perona-Malik algorithm [10] 11]

$$
\left\{\begin{array}{l}
\left.\frac{\partial I(\mathbf{X}(t), t)}{\partial t}=\operatorname{div}[|c(q)|) \cdot \nabla I(\mathbf{x}(t), t)\right] \\
I(\mathbf{x}(0), 0)=I_{0}
\end{array}\right.
$$

where $c(q)$ is the diffusion coefficient.

So, the speed term is defined in such a way that the curve proceeds rather fast in low gradient zones, while it wades through next to high gradient ones. This strategy allows the contour to propagate until it achieves the limits of the 


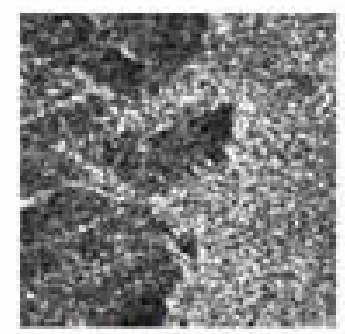

Criginal Image
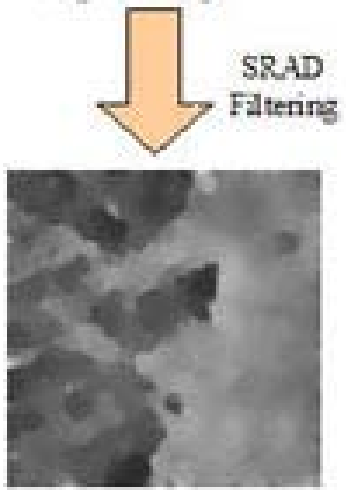

Filteredinage
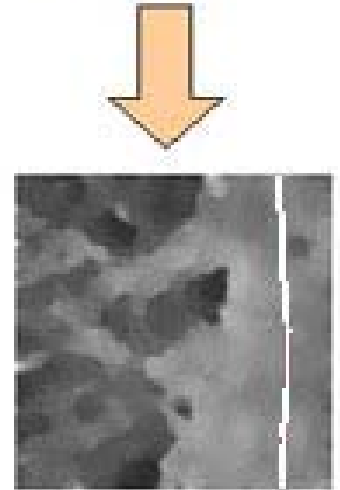

Initial Curve

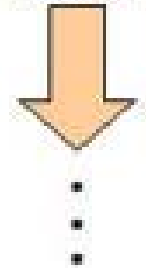

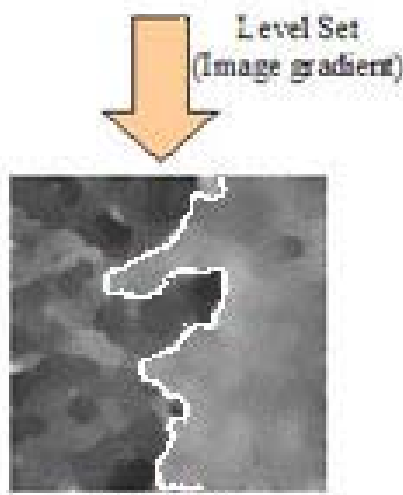

Fand Curve (Partial result)

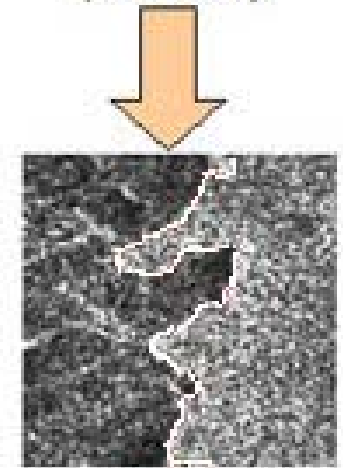

Initial Curve

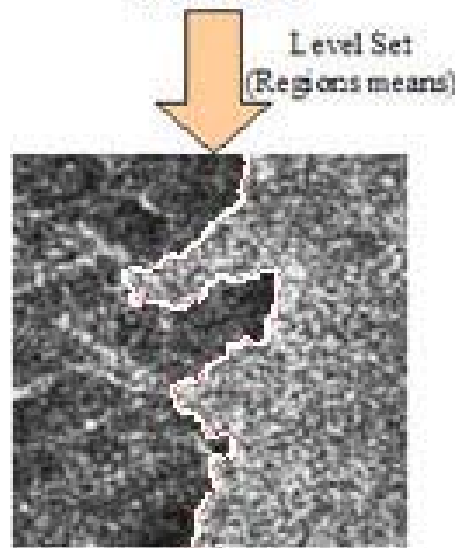

Find Curve

(Find Resul:)

Fig. 5. Final result of integrated procedure 
coastline in the image and then goes slowly close to those limits. In Figure 3 e Figure 4 two applications performed by gradient based speed are illustrated. Also in this case (a) and (b) represent a portion of initial curve selected (initial data for the numerical algorithm) while (c) and (d) show respectively the results (final boundaries) obtained when the convergence test is satisfied.

Unlike tests with average based speed, the same result is obtained from different initial curves.

\section{Integrated Procedure}

In order to obtain a more accurate result in the final position of the curve that identifies the coastline, an integration of both the procedures described above is proposed.

The implementation of Level Set with the speed based on regions means detects the coastline with more precision in terms of pixels, since the image is not dealt with filters for noise reduction. We observed that the best result is obtained by locating the initial curve as nearly as possible to the coastline, so that the final result depends on the position of the starting curve.

The implementation obtained by the speed based on image gradient is less accurate in terms of pixels than the previous one, because it works on the filtered image and not on the original one. However, this last approach is independent from the position of the initial curve.

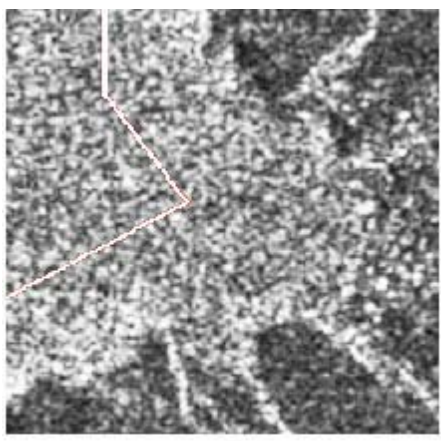

(a)

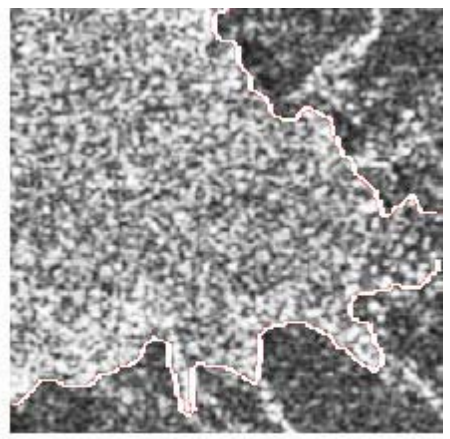

(b)

Fig. 6. Final result of integrated procedure

To obtain an automatic and accurate procedure, the two previous options have been integrated in the following way:

- The initial curve evolves applying the speed based on the image gradient to the image filtered by SRAD;

- The result is used as initial curve for the second application of level set to an original image by using the speed based on the means. 
The proposed procedure integrates the two speed functions improving the segmentation results. In fact, the combined procedure allows the coastline to be identified automatically and independently from the initial location of the curve. In Figure 5 we describe the application of the proposed integrated procedure.

The Figure 6 shows the results of the procedure applied to the original image related to Test $B$.

\section{Conclusion}

In this paper we have faced the problem of detecting the coastline from SAR image by the level set model. Two distinct speed evolution functions have been examined. The first, based on the mean intensities of the regions, does not need to reduce speckle noise; the other, based on the image gradient, takes into account the problem to filter speckle noise by the SRAD technique. We proposed an innovative procedure that combines the two speed functions improving the two individual approaches. In fact, the integrated procedure allows the segmentation result to be independent by the initial location of the curve and moreover, it automatically stops when the curve achieves the coastline.

Acknowledgments. The authors wish to thank the Consortium for Informatics and Telematics "Innova" of Matera, which has provided the PRI (Precision Images) images of ERS Mission.

\section{References}

[1] Sethian, J.A.: Level Set Methods and Fast Marching Methods. Cambridge University Press, Cambridge (1999)

[2] Sethian, J.A.: Evolution, Implementation and Application of Level Set and Fast Marching Methods for Advancing Fronts. Journal of Computional Physics 169, 503-555 (2001)

[3] Osher, S., Fedkiw, R.: Level Set Methods and Dynamic Implicit Surfaces. Springer, New York (2002)

[4] Lee, J.-S., Igor, J.: Coastline Detection and Tracing in SAR. Images IEEE Transaction on Geoscence and Remote Sensing 28, 662-668 (1999)

[5] Dellepiane, S., De Laurentiis, R., Giordano, F.: Coastline Extraction from Sar Images and a Method for the Evaluation of the Coastline Precision. Pattern Recognition Letters 25, 146-147 (2004)

[6] Kass, M., Witkin, A., Terzopoulos, D.: Snakes: Active Contour Models. International Journal Vision 1, 321-333 (1988)

[7] Germain, O., Refregier, P.: Edge Location in SAR Images: Performance of the Likehood Ratio Filter and Accuracy Improvement with an Active Contour Approach. IEEE Trans. Image Processing 10, 72-77 (2001)

[8] Chesnaud, C., Refregier, P., Boulet, V.: Statistical Region Snake-Based Segmentation Adapted to Different Physical Noise Models. IEEE Trans. Pattern Analysis and Machine Intelligence 21, 1145-1157 (1999) 
[9] Ben Ayed, I., Mitiche, A., Belhadj, Z.: Multiregion Level-Set Partitioning of Synthetic Aperture Radar Images. IEEE Trans. Pattern Analysis and Machine Intelligence 27, 793-800 (2005)

[10] Yu, Y., Acton, S.T.: Speckle Reducing Anisotropic Diffusion. IEEE Trans. on Image Processing 11, 1260-1270 (2002)

[11] Perona, P., Malik, J.: Scale Space and Edge Detection using Anisotropic Diffusion. IEEE Trans. Pattern Analysis and Machine Intelligence 12, 629-639 (1990) 